\title{
TESS Discovery of an Ultra-short-period Planet around the Nearby M Dwarf LHS 3844
}

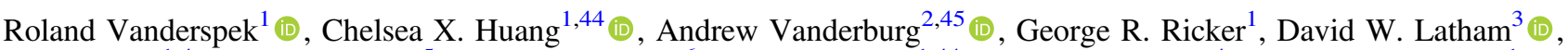
Sara Seager ${ }^{1,4}$, Joshua N. Winn ${ }^{5}$ (1) , Jon M. Jenkins ${ }^{6}$ (1) , Jennifer Burt ${ }^{1,44}$ (i), Jason Dittmann ${ }^{4}$ (1), Elisabeth Newton ${ }^{1}$ (1), Samuel N. Quinn ${ }^{3}$ (1) Avi Shporer ${ }^{1}$ (D), David Charbonneau ${ }^{3}$ (10), Jonathan Irwin $^{3}$, Kristo Ment ${ }^{3}$ (1) , Jennifer G. Winters ${ }^{3}$, Karen A. Collins ${ }^{3}$ (1), Phil Evans ${ }^{7}$, Tianjun Gan ${ }^{8}$, Rhodes Hart ${ }^{9}$, Eric L. N. Jensen ${ }^{10}$, John Kielkopf ${ }^{11}$, Shude Mao ${ }^{8}$ (1), William Waalkes ${ }^{12}$, François Bouchy ${ }^{13}$, Maxime Marmier ${ }^{13}$, Louise D. Nielsen ${ }^{13}$, Gaël Ottoni ${ }^{13}$, Francesco Pepe ${ }^{13}$, Damien Ségransan ${ }^{13}{ }^{\mathbb{1}}$, Stéphane Udry ${ }^{13}$, Todd Henry ${ }^{14}$, Leonardo A. Paredes ${ }^{15}$, Hodari-Sadiki James ${ }^{15}$, Rodrigo H. Hinojosa ${ }^{16}$, Michele L. Silverstein ${ }^{15}$ (10), Enric Palle ${ }^{17}$, Zachory Berta-Thompson ${ }^{12}$, Ian Crossfield ${ }^{1}$, Misty D. Davies ${ }^{6}$, Diana Dragomir ${ }^{1,47}$ (i), Michael Fausnaugh $^{1}$, Ana Glidden ${ }^{1}$ (1), Joshua Pepper ${ }^{18}$ (D), Edward H. Morgan ${ }^{1}$, Mark Rose ${ }^{19}$, Joseph D. Twicken ${ }^{20}$ (1),

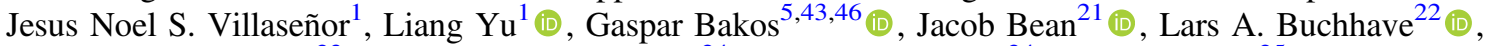
Jørgen Christensen-Dalsgaard ${ }^{23}$ (1), Jessie L. Christiansen ${ }^{24}$ (10, David R. Ciardi ${ }^{24}$, Mark Clampin ${ }^{25}$, Nathan De Lee ${ }^{26,27}$, Drake Deming $^{28}$, John Doty ${ }^{29}$, J. Garrett Jernigan ${ }^{30}$, Lisa Kaltenegger ${ }^{31}$, Jack J. Lissauer ${ }^{32}$, P. R. McCullough ${ }^{33}$, Norio Narita ${ }^{17,34,35,36,37 \text { (1) Martin Paegert }}{ }^{3}$ (1) , Andras Pal $^{38,39}$ (1), Stephen Rinehart ${ }^{40}$, Dimitar Sasselov ${ }^{3}$ (i), Bun'ei Sato ${ }^{41}$ (D), Alessandro Sozzetti ${ }^{42}$ (i), Keivan G. Stassun ${ }^{26}$ (i) , and Guillermo Torres ${ }^{3}$ (1)

${ }^{1}$ Department of Physics and Kavli Institute for Astrophysics and Space Science, Massachusetts Institute of Technology, Cambridge, MA 02139, USA

${ }^{2}$ Department of Astronomy, The University of Texas at Austin, Austin, TX 78712, USA

${ }^{3}$ Harvard-Smithsonian Center for Astrophysics, 60 Garden Street, Cambridge, MA 02138, USA

${ }^{4}$ Department of Earth and Planetary Sciences, Massachusetts Institute of Technology, Cambridge, MA 02139, USA

${ }^{5}$ Department of Astrophysical Sciences, Princeton University, Princeton, NJ 08544, USA

${ }^{6}$ NASA Ames Research Center, Moffett Field, CA 94035, USA

${ }^{7}$ El Sauce Observatory, Chile

${ }^{8}$ Department of Physics and Tsinghua Centre for Astrophysics, Tsinghua University, Beijing, People's Republic of China

${ }^{9}$ University of Southern Queensland, Toowoomba, Queensland 4350, Australia

${ }^{10}$ Department of Physics and Astronomy, Swarthmore College, Swarthmore, PA 19081, USA

${ }^{11}$ Department of Physics and Astronomy, University of Louisville, Louisville, KY 40292, USA

${ }^{12}$ Department of Astrophysical and Planetary Sciences, University of Colorado, Boulder, CO 80309, USA

${ }^{13}$ Observatoire de l'Université de Genève, 51 chemin des Maillettes, 1290 Versoix, Switzerland

${ }^{14}$ RECONS Institute, Chambersburg, PA 17201, USA

${ }^{15}$ Department of Physics and Astronomy, Georgia State University, Atlanta, GA 30302-4106, USA

${ }_{17}^{16}$ Cerro Tololo Inter-American Observatory, CTIO/AURA Inc., La Serena, Chile

${ }^{17}$ Instituto de Astrofisica de Canarias (IAC), E-38205 La Laguna, Tenerife, Spain

${ }^{18}$ Department of Physics, Lehigh University, Bethlehem, PA 18015, USA

${ }^{19}$ Leidos, Inc., Moffett Field, CA 94035, USA

${ }^{20}$ SETI Institute, Moffett Field, CA 94035, USA

${ }^{21}$ Department of Astronomy and Astrophysics, University of Chicago, Chicago, IL 60637, USA

22 DTU Space, National Space Institute, Technical University of Denmark, Elektrovej 328, DK-2800 Kgs. Lyngby, Denmark

${ }^{23}$ Stellar Astrophysics Centre, Department of Physics and Astronomy, Aarhus University, Denmark

${ }^{24}$ NASA Exoplanet Science Institute, Caltech/IPAC-NExScI, 1200 East California Boulevard, Pasadena, CA 91125, USA

${ }^{25}$ Sciences and Exploration Directorate, Goddard Space Flight Center, Greenbelt, MD 20771, USA

${ }^{26}$ Vanderbilt University, Department of Physics and Astronomy, Nashville, TN 37235, USA

${ }^{27}$ Department of Physics, Geology, and Engineering Technology, Northern Kentucky University, Highland Heights, KY 41099, USA

${ }^{28}$ University of Maryland at College Park, Department of Astronomy, College Park, MD 20742, USA

${ }^{29}$ Noqsi Aerospace, Ltd., 15 Blanchard Avenue, Billerica, MA 01821, USA

${ }^{30}$ Espace, Inc., 30 Lynn Avenue, Hull, MA 02045-2216, USA

${ }^{31}$ Carl Sagan Institute, Cornell University, Ithaca NY, 14853, USA

${ }^{32}$ Space Science and Astrobiology Division, NASA Ames Research Center, Moffett Field, CA 94035, USA

${ }^{33}$ Department of Physics and Astronomy, Johns Hopkins University, Baltimore, MD 21218, USA

${ }^{34}$ Department of Astronomy, University of Tokyo, Tokyo, Japan

${ }^{35}$ JST, PRESTO, Tokyo, Japan

${ }^{36}$ Astrobiology Center, 2-21-1 Osawa, Mitaka, Tokyo 181-8588, Japan

${ }^{37}$ National Astronomical Observatory of Japan, Tokyo, Japan

${ }^{38}$ Konkoly Observatory, Research Centre for Astronomy and Earth Sciences, Hungarian Academy of Sciences, Konkoly Thege Miklós út 15-17, H-1121 Budapest, Hungary

${ }^{39}$ Department of Astronomy, Loránd Eötvös University, Pázmány P. stny. 1/A, Budapest H-1117, Hungary

${ }^{40}$ Goddard Space Flight Center, Greenbelt, MD 20771, USA

${ }^{41}$ Department of Earth and Planetary Sciences, Tokyo Institute of Technology, Tokyo, Japan

${ }^{42}$ Osservatorio Astrofisico di Torino, INAF, Torino, Italy

${ }^{43}$ Konkoly Observatory, Hungary

Received 2018 September 17; revised 2018 December 17; accepted 2018 December 20; published 2019 January 25

\footnotetext{
44 Juan Carlos Torres Fellow.

45 NASA Sagan Fellow.

46 MTA Distinguished Guest Fellow.

${ }^{47}$ NASA Hubble Fellow.
} 


\begin{abstract}
Data from the newly commissioned Transiting Exoplanet Survey Satellite has revealed a "hot Earth" around LHS 3844, an M dwarf located 15 pc away. The planet has a radius of $1.303 \pm 0.022 R_{\oplus}$ and orbits the star every $11 \mathrm{hr}$. Although the existence of an atmosphere around such a strongly irradiated planet is questionable, the star is bright enough $(I=11.9, K=9.1)$ for this possibility to be investigated with transit and occultation spectroscopy. The star's brightness and the planet's short period will also facilitate the measurement of the planet's mass through Doppler spectroscopy.
\end{abstract}

Key words: planetary systems - planets and satellites: detection - stars: individual (LHS 3844, TIC 410153553)

\section{Introduction}

The Transiting Exoplanet Survey Satellite (TESS) is a NASA Explorer mission that was launched on 2018 April 18. The mission's primary objective is to discover hundreds of transiting planets smaller than Neptune, around stars bright enough for spectroscopic investigations of planetary masses and atmospheres (Ricker et al. 2015). Using four $10 \mathrm{~cm}$ refractive CCD cameras, TESS obtains optical images of a rectangular field spanning 2300 square degrees. The field is changed every 27.4 days (two spacecraft orbits), allowing the survey to cover most of the sky in 2 yr. TESS is a wider-field, brighter-star successor to the successful space-based transit surveys CoRoT (Baglin et al. 2006; Auvergne et al. 2009) and Kepler (Borucki et al. 2010).

Another way in which TESS differs from the previous space missions is that $\mathrm{M}$ dwarfs constitute a larger fraction of the stars being searched, mainly because of a redder observing bandpass (600-1000 nm). Compared to solar-type stars, M dwarfs are advantageous for transit surveys because the signals are larger for a given planet size, and because the transits of planets in the "habitable zone" are geometrically more likely and repeat more frequently (see, e.g., Gould et al. 2003; Charbonneau \& Deming 2007; Latham 2012). We also know that close-orbiting planets are very common around $\mathrm{M}$ dwarfs, based on results from the Kepler survey (Dressing \& Charbonneau 2015; Muirhead et al. 2015). By focusing on nearby $M$ dwarfs, the pioneering groundbased transit surveys MEarth and TRAPPIST have discovered four of the most remarkable planetary systems known today: GJ 1214 (Charbonneau et al. 2009), GJ 1132 (Berta-Thompson et al. 2015), LHS 1140 (Dittmann et al. 2017a; Ment et al. 2018), and TRAPPIST-1 (Gillon et al. 2016).

Simulations have shown that TESS should be capable of detecting hundreds of planets around nearby $\mathrm{M}$ dwarfs (Sullivan et al. 2015; Bouma et al. 2017; Ballard 2018; Barclay et al. 2018; Huang et al. 2018; Muirhead et al. 2018). Here, we report the first such detection, based on data from the first month of the survey. The planet has a radius of $1.32 \pm 0.02 R_{\oplus}$, and orbits the M dwarf LHS 3844 every $11 \mathrm{hr}$. The star, located 15 parsecs away, has a mass and radius that are about $15 \%$ and $19 \%$ of the Sun's values. The proximity and brightness of the star make this system a good candidate for follow-up Doppler and atmospheric spectroscopy.

This Letter is organized as follows. Section 2 presents the data from TESS along with follow-up observations with ground-based telescopes. Section 3 describes our method for determining the system parameters. This section also explains why the transit-like signal is very likely to represent a true planet and not an eclipsing binary or other types of "false positives." Section 4 compares LHS 3844b with the other known transiting planets, and discusses some possibilities for follow-up observations.

\section{Observations and Data Analysis}

\subsection{TESS}

TESS observed LHS 3844 between 2018 July 25 and August 22 , in the first of 26 sectors of the two-year survey. The star appeared in CCD 2 of Camera 3. The CCDs produce images every $2 \mathrm{~s}$, which are summed on board the spacecraft into images with an effective exposure time of 30 minutes. In addition, 2 minute images are prepared for subarrays surrounding preselected target stars, which are chosen primarily for the ease of detecting transiting planets. LHS 3844 was prioritized for 2 minute observations on account of its brightness in the TESS bandpass $(T=11.877)$, small stellar radius, and relative isolation from nearby stars (Muirhead et al. 2018; Stassun et al. 2018). LHS 3844 will not be observed again in the TESS primary mission.

The 2 minute data consist of 11 by 11 pixel subarrays. They were reduced with the Science Processing Operations Center (SPOC) pipeline, originally developed for the Kepler mission at the NASA Ames Research Center (Jenkins 2015; Jenkins et al. 2016). For LHS 3844, the signal-to-noise ratio of the transit signals was 32.4, using the definition of Twicken et al. (2018). The 30 minute data were analyzed independently with the MIT Quick Look Pipeline (C. X. Huang et al. 2018, in preparation). A transit search with the Box Least Square algorithm (BLS; Kovács et al. 2002) led to a detection with a signal-to-noise ratio of 31.6 , using the definition of Hartman \& Bakos (2016).

For subsequent analysis, we used the 2 minute Pre-search Data Conditioning light curve from the SPOC pipeline (Stumpe et al. 2012), which was extracted from the photometric aperture depicted in the lower right panel of Figure 1. The resulting light curve is shown in the top panel of Figure 2. To filter out lowfrequency variations, we fitted a basis spline to the light curve (excluding the transits and $3 \sigma$ outliers) and divided the light curve by the best-fit spline. The result is shown in the second panel of Figure 2. The interruption in the middle of the time series occurred when the spacecraft was at perigee, when it reorients and downlinks the data. There was also a 2 day interval when the data were compromised by abnormally unstable spacecraft pointing. In addition, we omitted the data collected in the vicinity of "momentum dumps," when the thrusters are used to reduce the speed of the spacecraft reaction wheels. These lasted 10-15 minutes and took place every 2.5 days.

\subsection{Ground-based Photometry}

LHS 3844 was observed by the ground-based MEarth-South telescope array as part of normal survey operations (Irwin et al. 2015; Dittmann et al. 2017b). A total of 1935 photometric observations were made between 2016 January 10 and 2018 


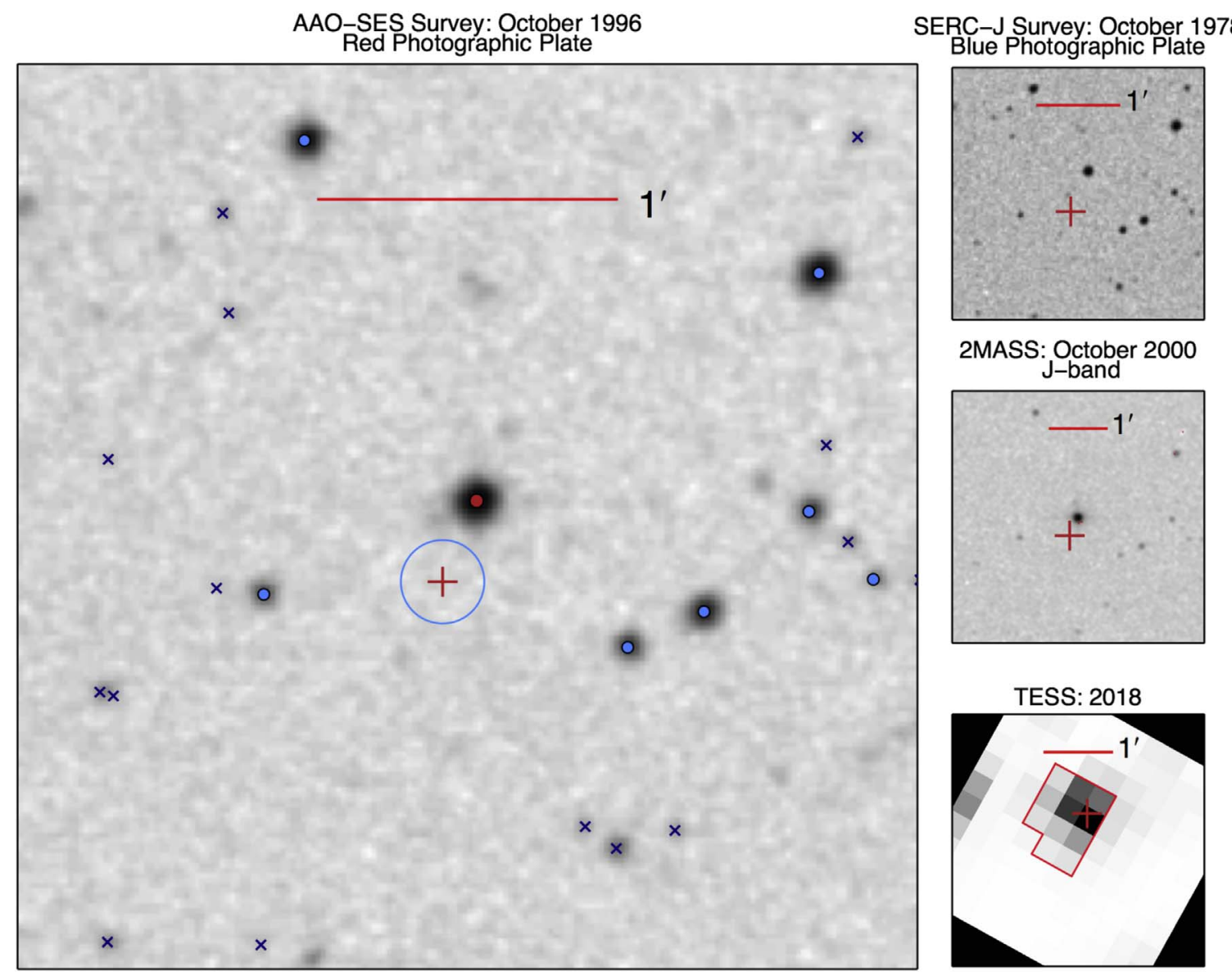

Figure 1. Images of the field surrounding LHS 3844. Left: from the Anglo-Australian Observatory Second Epoch Survey, obtained with a red-sensitive photographic emulsion in 1996. The red point is the location of LHS 3844 in this image, and the red cross indicates the current position. The blue points are stars that are bright enough to potentially be the source of the transit signal, while blue crosses are stars that are too faint. The blue circle shows the $10 \sigma$ upper limit on the motion of the center of light during transits. The lack of motion rules out the possibility that any of the surrounding stars is the source of the transit signal. Upper right: from the Science and Engineering Research Council J survey, obtained with a blue-sensitive photographic emulsion in 1978. Middle right: from the Two Micron All Sky Survey (2MASS) in $J$-band. Lower right: summed TESS image.

August 25. No transits had been detected prior to the TESS detection, but when the data were revisited, a BLS search identified a signal with a period and amplitude consistent with the TESS signal (Figure 2). The MEarth data (Figure 3) also revealed the stellar rotation period to be $128 \pm 24$ days, based on the least-squares periodogram of Irwin et al. (2006) as implemented by Newton et al. $(2016,2018){ }^{48}$

Additional ground-based transit observations were performed as part of the TESS Follow-up Observing Program (TFOP). A full transit was observed on UT 2018 September 06 in the $I_{C}$ band, using the El Sauce Observatory Planewave CDK14 telescope located in El Sauce, Chile. Five more transits were observed in the Sloan $i^{\prime}$ band using telescopes at the Cerro Tololo International Observatory (CTIO) node of the Las Cumbres Observatory $\left(\mathrm{LCO}^{49}\right)$ robotic telescope network (Brown et al. 2013). The transit of UT 2018 September 08 was observed with a $0.4 \mathrm{~m}$ telescope, and the transits of UT 2018 September 08, 09, 10, and 16 were observed with a $1.0 \mathrm{~m}$ telescope. The data are shown in the lower panels of

\footnotetext{
$\overline{48}$ Although Newton et al. (2018) did not detect rotational modulation, subsequent data have allowed for a "Grade B" detection, in the rating system described in that work.

49 https://lco.global
}

Figure 2. Together, they confirm the fading events are occurring and localize the source to within $2^{\prime \prime}$ of LHS 3844.

\subsection{High-resolution Spectroscopy}

We obtained optical spectra on UT 2018 June $18^{50}$ and September 08 using the CTIO HIgh ResolutiON (CHIRON) spectrograph on the $1.5 \mathrm{~m}$ telescope of the Cerro Tololo InterAmerican Observatory Small and Moderate Aperture Research Telescope System (Tokovinin et al. 2013). We used the image slicer mode, giving a resolution of about 80,000 . The signal-tonoise ratio of each spectrum is about 7 , and the spectral range is 411-877 nm. The first observation was a pair of 30 minute exposures centered at an orbital phase of 0.355 . The second observation comprised three 30 minute exposures centered at phase 0.880 . The data were analyzed as described by Winters et al. (2018b), using a spectrum of Barnard's Star as a template. The spectra show no evidence of additional lines from a stellar companion, no sign of rotational broadening, no detectable $\mathrm{H} \alpha$ emission, and no radial-velocity variation.

Additional spectroscopy was performed with the CORALIE spectrograph (Queloz et al. 2000; Pepe et al. 2017) on the

\footnotetext{
50 The spectra on UT 2018 June 18 were obtained as part of the program described by Winters et al. (2018a), before TESS started observations.
} 

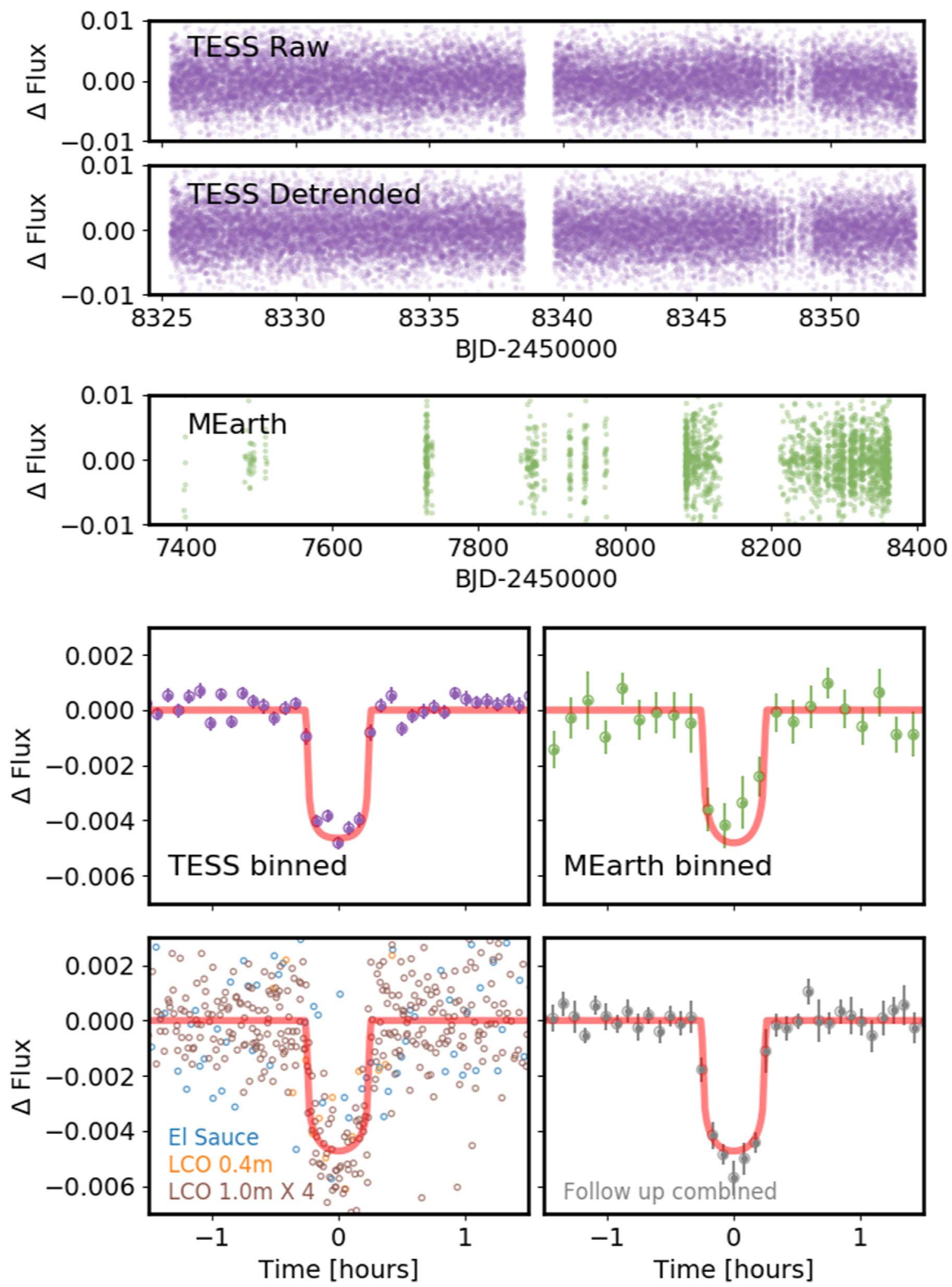

Figure 2. Light curves of LHS 3844. The top two panels show the TESS data, before and after high-pass filtering. The middle panel is from the MEarth Observatory, after correcting for systematics. The lower grid of four panels are phase-folded light curves, along with the best-fitting transit model. The TESS data points with error bars represent 5 minute averages. The MEarth data points represent 8 minute averages. The bottom two panels show data from the TESS Follow-up Observing Program, both the original data and 5 minute averages.

Swiss Euler $1.2 \mathrm{~m}$ telescope at La Silla Observatory in Chile. Spectra were obtained on UT 2018 September 10 and 11, at phases 0.211 and 0.645 , near the expected radial-velocity extrema. Radial-velocity calibration was performed with a
Fabry-Pérot device. With exposure times of 45 and 60 minutes, the signal-to-noise ratio per pixel was about 3 in the vicinity of $600 \mathrm{~nm}$. Cross-correlations were performed with a weighted M2 binary mask from which telluric and interstellar lines were 


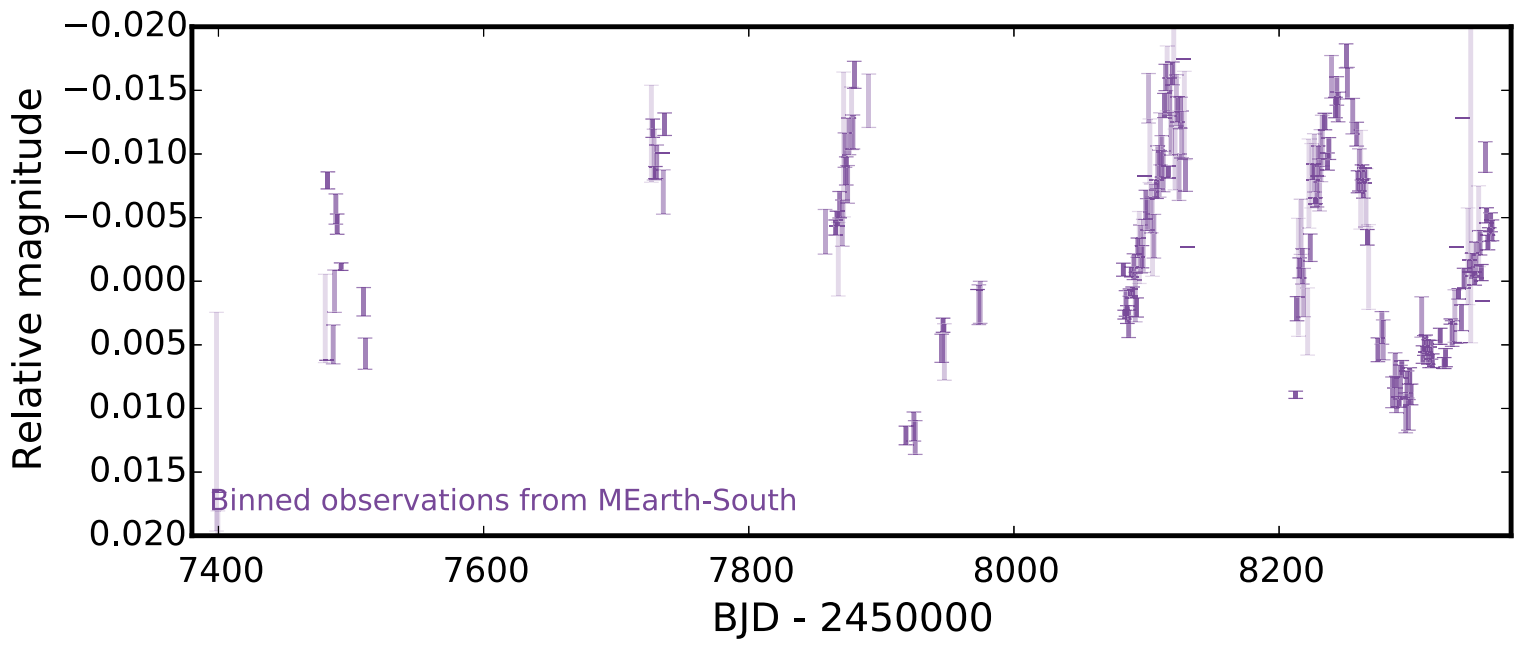

Figure 3. Long-term photometric monitoring of LHS 3844 by the MEarth Observatory. One-day averages are plotted, with error bars representing the standard error of the mean. Data points with higher precision are plotted with more opaque symbols. Based on the sinusoidal modulation observed in the most recent data, the rotation period is approximately 128 days.

removed (Pepe et al. 2002). Only a single peak was detected. The difference in radial velocities was $60 \pm 110 \mathrm{~m} \mathrm{~s}^{-1}$, i.e., not statistically significant.

To place an upper limit on the radial-velocity variation using both data sets (see Table 1), we fitted for the amplitude of a sinusoidal function with a period and phase specified by the TESS transit signal. The free parameters were the amplitude $K$, and two additive constants representing the zero-points of the CHIRON and CORALIE velocity scales. The result was $K=-28_{-60}^{+64} \mathrm{~m} \mathrm{~s}^{-1}$, which can be interpreted as a $3 \sigma$ upper limit of $0.96 M_{\text {Jup }}$ on the mass of the transiting object.

\section{Analysis \\ 3.1. Stellar Parameters}

Using an empirical relationship between mass and $K_{s}$-band absolute magnitude (Benedict et al. 2016), and the parallax from Data Release 2 of the Gaia mission (Gaia Collaboration et al. 2016, 2018; Lindegren et al. 2018), the mass of LHS 3844 is $0.151 \pm 0.014 M_{\odot}$. The uncertainty is dominated by the scatter in the mass $-K_{s}$ relation. Based on this mass determination, and the empirical mass-radius relationship of Boyajian et al. (2012), the stellar radius is $0.188 \pm 0.01 R_{\odot}$. These results are consistent with the empirical relationship between radius and absolute $K_{s}$ magnitude presented by Mann et al. (2015), which gives $0.189 \pm 0.006 R_{\odot}$. The bolometric luminosity is $(2.72 \pm 0.4) \times 10^{-3} L_{\odot}$, based on the observed $V$ and $J$ magnitudes and the bolometric correction from Table 3 of Mann et al. (2015). Based on these determinations of $R_{\star}$ and $L_{\star}$, the Stefan-Boltzmann law gives an effective temperature of $3036 \pm 77 \mathrm{~K}$. The spectral type is M4.5 or M5, based on a comparison with MEarth survey stars of known spectral types on a color-magnitude diagram (Gaia $G$ versus $H-K_{s}$ ).

As a check on the preceding calculations, we fitted stellar atmosphere models to the spectral energy distribution, based on the apparent magnitudes and the Gaia parallax, including the correction proposed by Stassun \& Torres (2018). This yielded a luminosity of $(2.56 \pm 0.4) \times 10^{-3} L_{\odot}$, an effective temperature of $2900 \pm 75 \mathrm{~K}$, and stellar radius of $0.201 \pm 0.012 R_{\odot}$. These values are all consistent with the stellar parameters derived above.
Table 1

Radial Velocities of LHS 3844

\begin{tabular}{lcccc}
\hline \hline BJD & $\begin{array}{c}\text { Orbital Phase } \\
\left(\mathrm{km} \mathrm{s}^{-1}\right)\end{array}$ & $\begin{array}{c}\mathrm{RV} \\
\left(\mathrm{km} \mathrm{s}^{-1}\right)\end{array}$ & $\sigma_{\mathrm{RV}}$ & Instrument \\
\hline 2458287.9183 & 0.33 & -10.626 & 0.056 & CHIRON \\
2458287.9393 & 0.375 & -10.667 & 0.108 & CHIRON \\
2458369.6266 & 0.833 & -10.719 & 0.031 & CHIRON \\
2458369.6476 & 0.878 & -10.732 & 0.046 & CHIRON \\
2458369.6686 & 0.924 & -10.724 & 0.048 & CHIRON \\
2458371.653268 & 0.211 & -10.600 & 0.090 & CORALIE \\
2458372.780028 & 0.645 & -10.540 & 0.068 & CORALIE \\
\hline
\end{tabular}

Note. The $100 \mathrm{~m} \mathrm{~s}^{-1}$ difference between the average CHIRON and CORALIE velocities is due to differences in the instrumental zero-points, and is not indicative of velocity variation.

\subsection{Light-curve Modeling}

We jointly analyzed the light curves from TESS, MEarth, and TFOP, using the formalism of Mandel \& Agol (2002) as implemented by Kreidberg (2015; batman). We assumed the orbit to be circular and used a quadratic limb-darkening law allowing the coefficients to be free. Because of the differing bandpasses of TESS, MEarth, and the TFOP instruments, each data set was allowed to have different values for the limbdarkening parameters. We imposed a prior constraint on the mean stellar density based on the results of Section 3.1. The model was evaluated with a 0.4 minute sampling and averaged as appropriate before comparing with the data. We used the emcee Markov Chain Monte Carlo code of Foreman-Mackey et al. (2013) to determine the posterior distributions for all the model parameters. The results are given in Table 2. Figure 2 shows the best-fitting model. The best-fit planet radii is $1.32 \pm 0.02 R_{\oplus}$ indicating that it is likely to be rocky (see, e.g., Rogers 2015, Wolfgang et al. 2016).

We also performed a fit to the TESS data only, without any prior constraint on the mean stellar density, in order to allow for a consistency check between the two density determinations. Based on the stellar parameters derived in Section 3.1, the mean density is $31.36 \pm 0.23 \mathrm{~g} \mathrm{~cm}^{-3}$, while the light-curve solution gives $30.0_{-2.8}^{+7.4} \mathrm{~g} \mathrm{~cm}^{-3}$. The agreement between these 
Table 2

Stellar and Planet Parameters for LHS 3844

\begin{tabular}{|c|c|c|}
\hline Parameter & Value & Source \\
\hline \multicolumn{3}{|l|}{ Catalog information } \\
\hline R.A. (h:m:s) & 22:41:59.089 & Gaia DR2 \\
\hline Decl. (d:m:s) & $-69: 10: 19.59$ & Gaia DR2 \\
\hline Epoch & 2015.5 & Gaia DR2 \\
\hline Parallax (mas) & $67.155 \pm 0.051$ & Gaia DR2 \\
\hline$\mu_{\text {R.A. }}\left(\operatorname{mas} \mathrm{yr}^{-1}\right)$ & $334.357 \pm 0.083$ & Gaia DR2 \\
\hline$\mu_{\text {Decl. }}\left(\right.$ mas yr $\left.^{-1}\right)$ & $-726.974 \pm 0.086$ & Gaia DR2 \\
\hline Gaia DR2 ID & 6385548541499112448 & \\
\hline TIC ID & 410153553 & \\
\hline LHS ID & 3844 & \\
\hline TOI ID & 136 & \\
\hline \multicolumn{3}{|l|}{ Photometric properties } \\
\hline TESS (mag) & 11.877 & TIC V7 \\
\hline Gaia (mag) & 13.393 & Gaia DR2 \\
\hline Gaia RP (mag) & 12.052 & Gaia DR2 \\
\hline Gaia BP (mag) & 15.451 & Gaia DR2 \\
\hline$V_{J}(\mathrm{mag})$ & $15.26 \pm 0.03$ & RECONS $^{\mathrm{a}}$ \\
\hline$R_{K C}(\mathrm{mag})$ & $13.74 \pm 0.02$ & RECONS $^{\mathrm{a}}$ \\
\hline$I_{K C}(\mathrm{mag})$ & $11.88 \pm 0.02$ & RECONS $^{\mathrm{a}}$ \\
\hline$J(\mathrm{mag})$ & $10.046 \pm 0.023$ & 2MASS \\
\hline$H(\mathrm{mag})$ & $9.477 \pm 0.023$ & 2MASS \\
\hline$K_{s}(\mathrm{mag})$ & $9.145 \pm 0.023$ & 2MASS \\
\hline \multicolumn{3}{|l|}{ Derived properties } \\
\hline$M_{\star}\left(M_{\odot}\right)$ & $0.151 \pm 0.014$ & $\begin{array}{l}\text { Parallax +Benedict } \\
\text { et al. }(2016)^{\mathrm{b}}\end{array}$ \\
\hline$R_{\star}\left(R_{\odot}\right)$ & $0.189 \pm 0.006$ & $\begin{array}{l}\text { Parallax +Mann } \\
\text { et al. }(2015)^{\mathrm{c}}\end{array}$ \\
\hline $\log g_{\star}(\mathrm{cgs})$ & $5.06 \pm 0.01$ & $\begin{array}{l}\text { empirical relation } \\
+\mathrm{LC}^{\mathrm{d}}\end{array}$ \\
\hline$L_{\star}\left(L_{\odot}\right)$ & $0.00272 \pm 0.0004$ & Mann et al. (2015) \\
\hline$T_{\text {eff } \star}(\mathrm{K})^{\mathrm{e}}$ & $3036 \pm 77$ & 3 \\
\hline$M_{V}(\mathrm{mag})$ & $14.39 \pm 0.02$ & Parallax \\
\hline$M_{K}(\mathrm{mag})$ & $8.272 \pm 0.015$ & Parallax \\
\hline Distance $(\mathrm{pc})$ & $14.9 \pm 0.01$ & Parallax \\
\hline$\rho_{\star}\left(\mathrm{g} \mathrm{cm}^{-3}\right)$ & $31.73 \pm 0.39$ & $\begin{array}{c}\text { empirical relation } \\
+\mathrm{LC}^{\mathrm{d}}\end{array}$ \\
\hline \multicolumn{3}{|l|}{ Light-curve parameters } \\
\hline$P$ (days) & $0.46292913 \pm 0.0000019$ & \\
\hline$T_{c}(\mathrm{BJD}-2457000)^{\mathrm{b}}$ & $1325.72558 \pm 0.00025$ & \\
\hline$T_{14}$ (minutes) $^{\mathrm{b}}$ & $31.27 \pm 0.28$ & \\
\hline$T_{12}=T_{34}{\text { (minutes })^{\mathrm{b}}}$ & $3.73_{-0.7}^{+0.4}$ & \\
\hline$a / R_{\star}$ & $7.109 \pm 0.029$ & \\
\hline$R_{p} / R_{\star}$ & $0.0635 \pm 0.0009$ & \\
\hline$b \equiv a \cos i / R_{\star}$ & $0.186 \pm 0.064$ & \\
\hline$i$ (degree) & $88.50 \pm 0.51$ & \\
\hline \multicolumn{3}{|l|}{ Limb-darkening coefficients } \\
\hline$c_{1}$, MEarth (linear term) & $0.15_{-0.10}^{+0.16}$ & \\
\hline$c_{2}$, MEarth (quadratic term) & $0.29 \pm 0.20$ & \\
\hline$c_{1}, T E S S$ & $0.17_{-0.11}^{+0.14}$ & \\
\hline$c_{2}, T E S S$ & $0.26_{-0.19}^{+0.22}$ & \\
\hline$c_{1}, i$ & $0.64_{-0.14}^{+0.12}$ & \\
\hline$c_{2}, i$ & $0.18_{-0.12}^{+0.18}$ & \\
\hline \multicolumn{3}{|l|}{ Planetary parameters } \\
\hline$R_{p}\left(R_{\oplus}\right)$ & $1.303 \pm 0.022$ & \\
\hline$a(\mathrm{au})$ & $0.00622 \pm 0.00017$ & \\
\hline$T_{\mathrm{eq}}(\mathrm{K})$ & $805 \pm 20$ & \\
\hline$\left\langle F_{j}\right\rangle\left(10^{9} \mathrm{erg} \mathrm{s}^{-1} \mathrm{~cm}^{-2}\right)$ & $0.0954 \pm 0.00070$ & \\
\hline
\end{tabular}

Notes.

a The optical photometry is from the RECONS survey, and was measured according to the procedures described in Jao et al. (2005) and Winters et al. (2015).

${ }^{b}$ We adopted the error bar based on the scatter in the empirical relations described by Benedict et al. (2016).

${ }^{\mathrm{c}}$ We adopted the error bar based on the scatter in the empirical relations described by Mann et al. (2015).

${ }^{\mathrm{d}}$ We fitted the transit light curves with a prior constraint on the stellar mass and radius derived from Gaia and broadband photometry.

${ }^{\mathrm{e}}$ The effective temperature was determined from the bolometric luminosity and the stellar radius. two results is a sign that the transit signal is from a planet, and is not an astrophysical false positive. A related point is that the ratio $\tau / T$ between the ingress/egress duration and the total duration is $0.11_{-0.02}^{+0.01}$, and less than 0.14 with $99 \%$ confidence. This information is used in Section 3.4 to help rule out the possibility that the fading events are from an unresolved eclipsing binary. In general, $\tau / T \gtrsim R_{p} / R_{\star}$, even when the photometric signal includes the constant light from an unresolved star (Morris et al. 2018).

\subsection{Photocenter Motion}

Many transit-like signals turn out to be from eclipsing binaries that are nearly along the same line of sight as the intended target star, such that the light from the binary is blended together with the constant light of the intended target star. These cases can often be recognized by measuring any motion of the center of light ("centroid") associated with the fading events (Wu et al. 2010). To do so, we modeled the time series of the $X$ and $Y$ coordinates of the center of light in the TESS images as though they were light curves, after removing long-timescale trends by fitting out a cubic spline. Based on the fitted depths of the "centroid transits" we were able to put $3 \sigma$ upper limits on centroid shifts of $\Delta X<2 \times 10^{-4}$ and $\Delta Y<6 \times 10^{-4}$ pixels, corresponding to 4.4 and 13.2 mas. Thus, there is no evidence for photocenter motion.

\subsection{Possible False Positives}

As mentioned previously, not all transit-like signals are from transiting planets. Below, we consider the usual alternatives to a transiting planet, and explain how the available data render them very unlikely.

1. The signal is an instrumental artifact. This is ruled out by the detection of the transit signals with ground-based telescopes (Section 2).

2. LHS 3844 is an eclipsing binary star. This is ruled out by the upper limit on radial-velocity variations, corresponding to a secondary mass of $0.96 M_{\text {Jup }}$ (Section 2.3). In addition, the absence of detectable phase variations in the TESS light curve requires that any companion be substellar. An $80 M_{\text {Jup }}$ companion would have produced ellipsoidal variations of order $0.1 \%$ (see, e.g., Shporer 2017), which can be excluded.

3. Light from a distant eclipsing binary, or a distant star with a transiting planet, is blended with that of LHS 3844. We can rule out this possibility thanks to the star's high proper motion $\left(800\right.$ mas $\left.\mathrm{yr}^{-1}\right)$. Because the star moves quickly relative to background stars, images from previous widefield surveys allow us to check for faint stars along the current line of sight. No sources are detected within 6 mag of LHS 3844, the brightness level that would be required to produce $0.4 \%$ flux dips. In addition, the ground-based observations require the fading source to be within $2^{\prime \prime}$ of LHS 3844 (Section 2.2), and the TESS images reveal no detectable motion of the stellar image during transits (Section 3.3).

4. LHS 3844 is physically associated with an eclipsing binary star. The light-curve analysis (Section 3.2) requires the eclipsing object to be smaller than $16 \%$ of the size of the eclipsed star. Since the spectrum is that of 

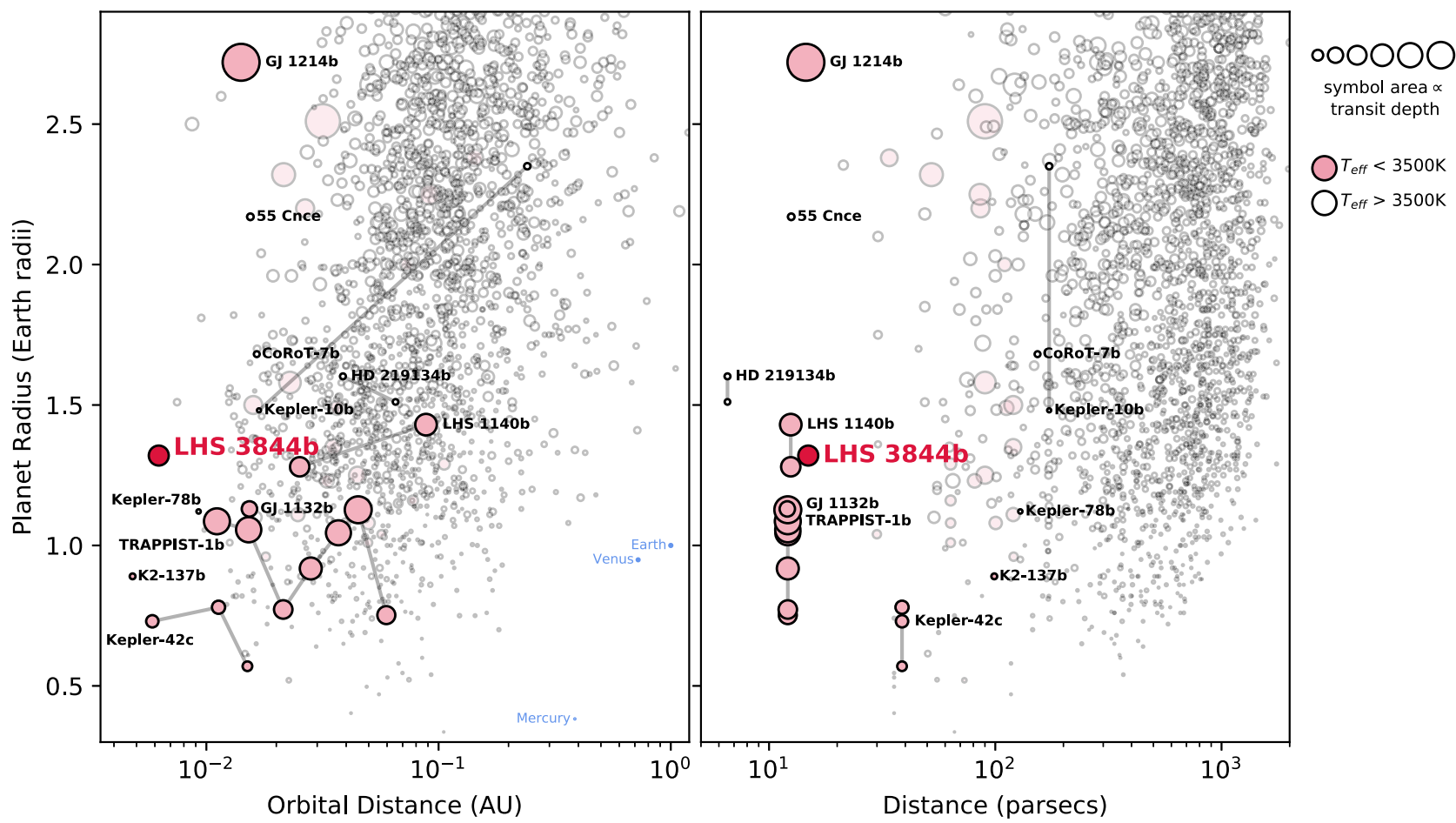

Figure 4. LHS $3844 \mathrm{~b}$ in the context of other known exoplanets. Left: planet radius and orbital distance for confirmed transiting planets. Right: planet radius and distance from Earth. The area of each circle is proportional to the transit depth of the planet. Pink circles represent host stars with $T_{\text {eff }}<3500 \mathrm{~K}$. Planets in the same system are connected by lines. Based on data from the NASA Exoplanet Archive, accessed on 2018 September 13 (https://exoplanetarchive.ipac.caltech.edu/cgibin/TblView/nph-tblView?app=ExoTbls\&config=planets).

an M4-5 dwarf, any secondary star would need to be of that size or smaller, implying that the eclipsing object is smaller than $0.16 \times 0.19 R_{\odot}$ or $3.0 R_{\oplus}$. This rules out a stellar binary.

5. LHS 3844 is a binary star and the transiting planet is around the secondary star. The companion would have to be faint and close to LHS 3844 in order to escape detection by Gaia (Rizzuto et al. 2018; Ziegler et al. 2018). Another indication that any secondary star needs to be faint is that the Gaia parallax and apparent magnitude are consistent with the properties of a single $\mathrm{M}$ dwarf. However, if the transiting planet is around such a faint companion, then the true transit depth must be less than about $2 \%$ in order for the transiting object to be smaller than $16 \%$ the size of the eclipsed star. Thus, in order to produce the $0.4 \%$ transit we observe, a secondary star would have to contribute at least $20 \%$ of the total flux in the TESS aperture while still escaping detection by Gaia and seeing-limited imaging.

Thus, almost all of these scenarios are ruled out, except for the possibility that the planet is actually orbiting a lowluminosity secondary star. This scenario seems contrived, and is a priori unlikely because of the low companion fraction for mid-M dwarfs (Winters et al. 2018b), the small parameter space for companions that could produce the transits we observe, the lower probability for an $\mathrm{M}$ dwarf to host a larger planet compared to a $1.3 R_{\oplus}$ planet (Berta et al. 2013; Mulders et al. 2015), and the tendency of selection effects to favor finding transits around primary stars (Bouma et al. 2018). Probably the only way to rule out this possibility, or more exotic scenarios, is through precise Doppler monitoring and adaptive optics imaging. The vespa code (Morton 2015), applied to LHS 3844, confirms that the false positive probabilities due to background eclipsing binaries and hierarchical eclipsing binaries are extremely low (it returns a false positive probability of order $10^{-16}$ ).

\section{Discussion}

LHS 3844b is one of the closest known planets, both in terms of its distance from the Earth and its distance from its host star (see Figure 4). It joins the small club of transiting planets around the Sun's nearest M dwarf neighbors, which also includes GJ 1214b (Charbonneau et al. 2009), GJ 1132b (Berta-Thompson et al. 2015), TRAPPIST-1 b-h (Gillon et al. 2016), and LHS 1140 b-c (Dittmann et al. 2017a; Ment et al. 2018). LHS 3844b is also the most easily studied example of an ultra-short-period (USP) planet, defined by the simple criterion $P<1$ day (Sanchis-Ojeda et al. 2014; Winn et al. 2018). It has the largest transit depth of any known sub-Jovian USP planet, and is closer to Earth than the other well-known systems CoRoT-7 (Léger et al. 2009), Kepler-10 (Batalha et al. 2011), Kepler-42 (Muirhead et al. 2012), and Kepler-78 (SanchisOjeda et al. 2013).

As such, LHS 3844b provides interesting opportunities for atmospheric characterization through transit and occultation (secondary eclipse) spectroscopy. With an equilibrium temperature of about $805 \mathrm{~K}$, and an orbital distance amounting to only 7.1 stellar radii, it is unclear what type of atmosphere the planet might have, if any. If the planet formed at or near this location, its primordial atmosphere could have been completely stripped away during the host star's youth, when it was much more luminous and chromospherically active. The observed radius function of the short-period Kepler planets has a dip at around $1.8 R_{\oplus}$ that has been interpreted as a consequence of 
atmospheric loss (Fulton et al. 2017; Fulton \& Petigura 2018; Van Eylen et al. 2018). Planets smaller than $1.8 R_{\oplus}$ seem to have lost their primordial hydrogen-helium atmospheres due to photoevaporation (Lopez \& Fortney 2013; Owen \& Wu 2013). With a radius of $1.32 R_{\oplus}$, we might expect LHS 3844 b to have suffered this process, too. In this case, transit spectroscopy would show no variation in the planetary radius with wavelength, although occultation spectroscopy could still be used to measure the emission spectrum of the planet's surface.

Indeed, of all the known planets smaller than $2 R_{\oplus}$, LHS $3844 \mathrm{~b}$ has perhaps the most readily detectable occultations. This is based on a ranking of the 907 planets in the NASA Exoplanet Archive by a crude signal-to-noise metric,

$$
\mathrm{S} / \mathrm{N} \propto \sqrt{F_{\star}} \frac{R_{p}^{2} T_{p}}{R_{\star}^{2} T_{\star}},
$$

which assumes that the star and planet are both radiating as blackbodies in the Rayleigh-Jeans limit. Here, $F_{\star}$ is the star's $K$-band flux, $T_{p}$ is the planet's equilibrium temperature, and $T_{\star}$ is the star's effective temperature. ${ }^{51}$ According to this metric, LHS 3844b ranks second, closely trailing HD 219134 b, which orbits a much brighter, but larger, star. Even then, LHS 3844b will likely be easier to observe than HD 219134 b thanks to its significantly deeper secondary eclipse, which should avoid observational systematic noise floors, and the planet's ultrashort period, which simplifies scheduling observations.

The ultra-short period will also facilitate the measurement of the planet's mass through Doppler spectroscopy. Short periods lead to stronger signals: assuming the planet's mass is $2.8 M_{\oplus}$, as it would be for a terrestrial composition, the expected semiamplitude of the Doppler signal is $8 \mathrm{~m} \mathrm{~s}^{-1}$, which is unusually high for a rocky planet. The orbital period is short enough for the signal to be measured in its entirety in just a few nights. The stellar rotation period is also 280 times longer than the orbital, allowing for a clear separation of timescales between the orbital motion and any spurious Doppler signals related to stellar activity.

The discovery of a potential rocky planet around a nearby $M$ dwarf during the first TESS observing sector suggests that the prospects for future discoveries are bright. It is worth remembering that $90 \%$ of the sky has not yet been surveyed by either TESS or Kepler.

We acknowledge the use of TESS Alert data, which is currently in a beta test phase, from the TESS Science Office. Funding for the TESS mission is provided by NASA's Science Mission directorate. This research has made use of the Exoplanet Follow-up Observation Program website, which is operated by the California Institute of Technology, under contract with the National Aeronautics and Space Administration under the Exoplanet Exploration Program. This work makes use of observations from the LCOGT network. Work by J.N.W. was partially supported by the Heising-Simons Foundation. This work has made use of data from the European Space Agency (ESA) mission Gaia (https://www. cosmos.esa.int/gaia), processed by the Gaia Data Processing and Analysis Consortium (DPAC, https://www.cosmos.esa. int/web/gaia/dpac/consortium). Funding for the DPAC has been provided by national institutions, in particular, the

\footnotetext{
${ }^{51}$ See also the work by Kempton et al. (2018).
}

institutions participating in the Gaia Multilateral Agreement. The MEarth team acknowledges funding from the David and Lucile Packard Fellowship for Science and Engineering (awarded to D.C.). This material is based on work supported by the National Science Foundation under grants AST0807690, AST-1109468, AST-1004488 (Alan T. Waterman Award), and AST-1616624. Acquisition of the CHIRON data was made possible through the support of a grant from the John Templeton Foundation. The opinions expressed in this publication are those of the authors and do not necessarily reflect the views of the John Templeton Foundation. J.A.D. acknowledges support by the Heising-Simons Foundation as a 51 Pegasi b postdoctoral fellow. E.R.N. is supported by an NSF Astronomy and Astrophysics Postdoctoral Fellowship under award AST-1602597. We thank the Geneva University and the Swiss National Science Foundation for their continuous support for the Euler telescope. This research has made use of the NASA Exoplanet Archive, which is operated by the California Institute of Technology, under contract with the National Aeronautics and Space Administration under the Exoplanet Exploration Program. C.X.H. acknowledges support from MIT's Kavli Institute as a Torres postdoctoral fellow. A.V.'s work was performed under contract with the California Institute of Technology/Jet Propulsion Laboratory funded by NASA through the Sagan Fellowship Program executed by the NASA Exoplanet Science Institute. Funding for the Stellar Astrophysics Centre is provided by The Danish National Research Foundation (Grant DNRF106). This work is partly supported by JSPS KAKENHI grant No. JP18H01265 and JST PRESTO grant No. JPMJPR1775. D.D. acknowledges support provided by NASA through Hubble Fellowship grant HSTHF2-51372.001-A awarded by the Space Telescope Science Institute, which is operated by the Association of Universities for Research in Astronomy, Inc., for NASA, under contract NAS5-26555.

Facilities: TESS, CTIO:1.5m (CHIRON), Euler $1.2 \mathrm{~m}$ (CORALIE), LCO:0.4m (SBIG), LCO:1.0m (Sinistro).

\section{ORCID iDs}

Roland Vanderspek (i) https://orcid.org/0000-0001-6763-6562 Chelsea X. Huang (i) https://orcid.org/0000-0003-0918-7484 Andrew Vanderburg (i) https://orcid.org/0000-00017246-5438

David W. Latham (1) https://orcid.org/0000-0001-9911-7388

Joshua N. Winn (D) https://orcid.org/0000-0002-4265-047X

Jon M. Jenkins (iD https://orcid.org/0000-0002-4715-9460 Jennifer Burt (ib https://orcid.org/0000-0002-0040-6815 Jason Dittmann (iD https://orcid.org/0000-0001-7730-2240 Elisabeth Newton (iD https://orcid.org/0000-0003-4150-841X Samuel N. Quinn (i) https://orcid.org/0000-0002-8964-8377 Avi Shporer (i) https://orcid.org/0000-0002-1836-3120 David Charbonneau (i) https://orcid.org/0000-00029003-484X

Kristo Ment (10) https://orcid.org/0000-0001-5847-9147

Karen A. Collins (iD https://orcid.org/0000-0001-6588-9574

Shude Mao (10) https:// orcid.org/0000-0001-8317-2788

Damien Ségransan (1) https://orcid.org/0000-0003-2355-8034

Michele L. Silverstein (1) https://orcid.org/0000-00032565-7909

Diana Dragomir (DD https://orcid.org/0000-0003-2313-467X

Ana Glidden (D) https://orcid.org/0000-0002-5322-2315

Joshua Pepper (iD https://orcid.org/0000-0002-3827-8417 
Joseph D. Twicken (1) https://orcid.org/0000-0002-6778-7552

Liang Yu (i) https://orcid.org/0000-0003-1667-5427

Gaspar Bakos (1) https://orcid.org/0000-0001-7204-6727

Jacob Bean (1) https://orcid.org/0000-0003-4733-6532

Lars A. Buchhave (1) https://orcid.org/0000-0003-1605-5666

Jørgen Christensen-Dalsgaard (i) https://orcid.org/0000-00015137-0966

Jessie L. Christiansen (1) https://orcid.org/0000-00028035-4778

Norio Narita (1) https://orcid.org/0000-0001-8511-2981

Martin Paegert 낭 https://orcid.org/0000-0001-8120-7457

Andras Pal (i) https://orcid.org/0000-0001-5449-2467

Dimitar Sasselov (ib) https://orcid.org/0000-0001-7014-1771

Bun'ei Sato (1) https://orcid.org/0000-0001-8033-5633

Alessandro Sozzetti 10 https://orcid.org/0000-0002-

7504-365X

Keivan G. Stassun (1) https://orcid.org/0000-0002-3481-9052

Guillermo Torres (1) https://orcid.org/0000-0002-5286-0251

\section{References}

Auvergne, M., Bodin, P., Boisnard, L., et al. 2009, A\&A, 506, 411

Baglin, A., Auvergne, M., Boisnard, L., et al. 2006, in COSPAR Scientific Assembly 36, Abstract \#3749

Ballard, S. 2018, arXiv:1801.04949

Barclay, T., Pepper, J., \& Quintana, E. V. 2018, ApJS, 239, 2

Batalha, N. M., Borucki, W. J., Bryson, S. T., et al. 2011, ApJ, 729, 27

Benedict, G. F., Henry, T. J., Franz, O. G., et al. 2016, AJ, 152, 141

Berta-Thompson, Z. K., Irwin, J., Charbonneau, D., et al. 2015, Natur, 527, 204

Berta, Z. K., Irwin, J., \& Charbonneau, D. 2013, ApJ, 775, 91

Borucki, W. J., Koch, D., Basri, G., et al. 2010, Sci, 327, 977

Bouma, L. G., Masuda, K., \& Winn, J. N. 2018, AJ, 155, 244

Bouma, L. G., Winn, J. N., Kosiarek, J., \& McCullough, P. R. 2017, arXiv: 1705.08891

Boyajian, T. S., von Braun, K., van Belle, G., et al. 2012, ApJ, 757, 112

Brown, T. M., Baliber, N., Bianco, F. B., et al. 2013, PASP, 125, 1031

Charbonneau, D., Berta, Z. K., Irwin, J., et al. 2009, Natur, 462, 891

Charbonneau, D., \& Deming, D. 2007, arXiv:0706.1047

Dittmann, J. A., Irwin, J. M., Charbonneau, D., et al. 2017a, Natur, 544, 333

Dittmann, J. A., Irwin, J. M., Charbonneau, D., Berta-Thompson, Z. K., \& Newton, E. R. 2017b, AJ, 154, 142

Dressing, C. D., \& Charbonneau, D. 2015, ApJ, 807, 45

Foreman-Mackey, D., Hogg, D. W., Lang, D., \& Goodman, J. 2013, PASP, 125,306

Fulton, B. J., \& Petigura, E. A. 2018, arXiv:1805.01453

Fulton, B. J., Petigura, E. A., Howard, A. W., et al. 2017, AJ, 154, 109

Gaia Collaboration, Brown, A. G. A., Vallenari, A., et al. 2018, A\&A, 616, A1

Gaia Collaboration, Prusti, T., de Bruijne, J. H. J., et al. 2016, A\&A, 595, A1

Gillon, M., Jehin, E., Lederer, S. M., et al. 2016, Natur, 533, 221

Gould, A., Pepper, J., \& DePoy, D. L. 2003, ApJ, 594, 533

Hartman, J. D., \& Bakos, G. Á. 2016, A\&C, 17, 1

Huang, C. X., Shporer, A., Dragomir, D., et al. 2018, arXiv:1807.11129
Irwin, J., Aigrain, S., Hodgkin, S., et al. 2006, MNRAS, 370, 954

Irwin, J. M., Berta-Thompson, Z. K., Charbonneau, D., et al. 2015, in 18th Cambridge Workshop on Cool Stars, Stellar Systems, and the Sun, ed. G. van Belle \& H. Harris, 767

Jao, W.-C., Henry, T. J., Subasavage, J. P., et al. 2005, AJ, 129, 1954

Jenkins, J. M. 2015, BAAS, 47, 6

Jenkins, J. M., Twicken, J. D., McCauliff, S., et al. 2016, Proc. SPIE, 9913, 99133E

Kempton, E. M.-R., Bean, J. L., Louie, D. R., et al. 2018, PASP, 130, 114401

Kovács, G., Zucker, S., \& Mazeh, T. 2002, A\&A, 391, 369

Kreidberg, L. 2015, PASP, 127, 1161

Latham, D. W. 2012, NewA, 56, 16

Léger, A., Rouan, D., Schneider, J., et al. 2009, A\&A, 506, 287

Lindegren, L., Hernández, J., Bombrun, A., et al. 2018, A\&A, 616, A2

Lopez, E. D., \& Fortney, J. J. 2013, ApJ, 776, 2

Mandel, K., \& Agol, E. 2002, ApJL, 580, L171

Mann, A. W., Feiden, G. A., Gaidos, E., Boyajian, T., \& von Braun, K. 2015, ApJ, 804, 64

Ment, K., Dittmann, J. A., Astudillo-Defru, N., et al. 2018, AJ, 157, 32

Morris, B. M., Agol, E., Hebb, L., \& Hawley, S. L. 2018, AJ, 156, 91

Morton, T. D. 2015, VESPA: False Positive Probabilities Calculator, Astrophysics Source Code Library, ascl:1503.011

Muirhead, P. S., Dressing, C. D., Mann, A. W., et al. 2018, AJ, 155, 180

Muirhead, P. S., Johnson, J. A., Apps, K., et al. 2012, ApJ, 747, 144

Muirhead, P. S., Mann, A. W., Vanderburg, A., et al. 2015, ApJ, 801, 18

Mulders, G. D., Pascucci, I., \& Apai, D. 2015, ApJ, 814, 130

Newton, E. R., Irwin, J., Charbonneau, D., Berta-Thompson, Z. K., \& Dittmann, J. A. 2016, ApJL, 821, L19

Newton, E. R., Mondrik, N., Irwin, J., Winters, J. G., \& Charbonneau, D. 2018, AJ, 156, 217

Owen, J. E., \& Wu, Y. 2013, ApJ, 775, 105

Pepe, F., Bouchy, F., Mayor, M., \& Udry, S. 2017, in Handbook of Exoplanets, ed. H. Deeg \& J. Belmonte (Cham: Springer), 190

Pepe, F., Mayor, M., Galland, F., et al. 2002, A\&A, 388, 632

Queloz, D., Mayor, M., Weber, L., et al. 2000, A\&A, 354, 99

Ricker, G. R., Winn, J. N., Vanderspek, R., et al. 2015, JATIS, 1, 014003

Rizzuto, A. C., Vanderburg, A., Mann, A. W., et al. 2018, AJ, 156, 195

Rogers, L. A. 2015, ApJ, 801, 41

Sanchis-Ojeda, R., Rappaport, S., Winn, J. N., et al. 2013, ApJ, 774, 54

Sanchis-Ojeda, R., Rappaport, S., Winn, J. N., et al. 2014, ApJ, 787, 47

Shporer, A. 2017, PASP, 129, 072001

Stassun, K. G., Oelkers, R. J., Pepper, J., et al. 2018, AJ, 156, 102

Stassun, K. G., \& Torres, G. 2018, ApJ, 862, 61

Stumpe, M. C., Smith, J. C., Van Cleve, J. E., et al. 2012, PASP, 124, 985

Sullivan, P. W., Winn, J. N., Berta-Thompson, Z. K., et al. 2015, ApJ, 809, 77

Tokovinin, A., Fischer, D. A., Bonati, M., et al. 2013, PASP, 125, 1336

Twicken, J. D., Catanzarite, J. H., Clarke, B. D., et al. 2018, PASP, 130, 064502

Van Eylen, V., Agentoft, C., Lundkvist, M. S., et al. 2018, MNRAS, 479, 4786

Winn, J. N., Sanchis-Ojeda, R., \& Rappaport, S. 2018, arXiv:1803.03303

Winters, J., Irwin, J., Newton, E., et al. 2018a, BAAS, 231, 334.06

Winters, J. G., Henry, T. J., Lurie, J. C., et al. 2015, AJ, 149, 5

Winters, J. G., Irwin, J., Newton, E. R., et al. 2018b, AJ, 155, 125

Wolfgang, A., Rogers, L. A., \& Ford, E. B. 2016, ApJ, 825, 19

Wu, H., Twicken, J. D., Tenenbaum, P., et al. 2010, Proc. SPIE, 7740, 774019

Ziegler, C., Law, N. M., Baranec, C., et al. 2018, AJ, 156, 259 